\title{
Tracking Length and Differential-Wavefront-Sensing Signals from Quadrant Photodiodes in Heterodyne Interferometers with Digital Phase-Locked-Loop Readout
}

\author{
Gerhard Heinzel@, ${ }^{*}$ Miguel Dovale Álvarez®, ${ }^{\dagger}$ Alvise Pizzella®, Nils Brause, and \\ Juan José Esteban Delgado \\ Max Planck Institute for Gravitational Physics (Albert Einstein Institute) and Institute for Gravitational Physics of \\ the Leibniz Universität Hannover, Callinstraße 38, 30167 Hannover, Germany
}

(Received 3 August 2020; revised 18 September 2020; accepted 8 October 2020; published 6 November 2020)

\begin{abstract}
We propose a method to track signals from quadrant photodiodes (QPDs) in heterodyne laser interferometers that employ digital phase-locked loops for phase readout. Instead of separately tracking the four segments from the QPD and then combining the results into length and differential-wavefront-sensing signals, this method employs a set of coupled tracking loops that operate directly on the combined length and angular signals. The benefits are an increased signal-to-noise ratio in the loops and the possibility of adapting the loop bandwidths to the differing dynamical behavior of the signals being tracked, which now correspond to physically meaningful observables. We demonstrate an improvement of up to $6 \mathrm{~dB}$ over single-segment tracking, which makes this scheme an attractive solution for applications in precision intersatellite laser interferometry in ultralow light conditions.
\end{abstract}

DOI: 10.1103/PhysRevApplied.14.054013

\section{INTRODUCTION}

Laser interferometry is a powerful method to measure tiny distance variations as changes of optical path lengths. When the optical path lengths cannot be kept constant to within a small fraction of a wavelength, heterodyne interferometry is frequently applied, i.e., by interfering two laser beams that have a finite frequency difference. The interference pattern is recorded with a photodiode, which produces a photocurrent with a sinusoidal component at the heterodyne frequency. Differential changes of optical path length are then converted into phase changes of that sinusoidal beat note, which are measured with a phasemeter instrument. Heterodyne laser interferometers have been employed with great success in space-based gravity missions [1,2], which require high-precision displacement sensors with a large dynamic range.

Several techniques exist to perform this phase measurement with subpicometer precision in the millihertz frequency band as required for applications in gravimetry, gradiometry, and gravitational-wave detection [3-12]. When the heterodyne frequency changes with time-as,

\footnotetext{
*gerhard.heinzel@aei.mpg.de

†miguel.dovale@aei.mpg.de
}

Published by the American Physical Society under the terms of the Creative Commons Attribution 4.0 International license. Further distribution of this work must maintain attribution to the author(s) and the published article's title, journal citation, and DOI. e.g., over interspacecraft optical links such as the laser interferometer space antenna (LISA) mission [13,14] or the Laser Ranging Interferometer (LRI) on GRACE Follow-On $[15,16]$ - the usual technique is to employ a digital-phase-locked-loop (DPLL) [7-12,17] architecture to track the amplitude, frequency, and phase of the beat note, even when the received beam is sensed at the subpicowatt power level due to the beam divergence over the large propagation distances typical of intersatellite laser links.

In many applications of laser interferometers, such as in gravitational reference sensors [18], spacecraft attitude control [19], or precision laser-beam pointing to remote spacecraft [20], it is beneficial or even essential to determine not only the relative optical-path-length change between the two interfering beams but also the angle between their wavefronts, since the latter is a very sensitive measurement of misalignments in the optical system. The standard technique to achieve this angular measurement is differential wavefront sensing (DWS) [21,22], which uses a quadrant photodiode (QPD) to detect the interference pattern. The average phase over the four segments represents the length signal, while the difference between left and right or top and bottom represents horizontal and vertical misalignments, respectively (see Fig. 1).

In previous implementations, the phase measurement has been applied separately to each segment of the diode and the results then combined. This paper describes a method to track the phases of the beat notes from the four segments of a quadrant photodiode with DPLLs. In Sec. II, 


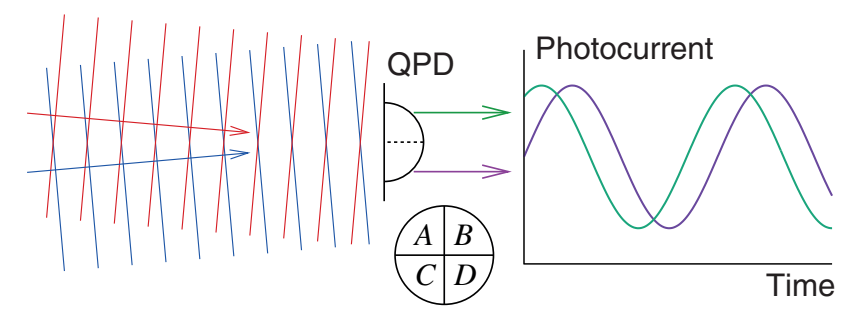

FIG. 1. An illustration of the DWS method. Two waves impinge on the surface of a quadrant photodiode (QPD) at an angle. The upper and lower segments of the QPD thus measure different phase offsets between the interfering wavefronts. In the case of heterodyne interference, this angular misalignment between wavefronts manifests as shifts of the phase of the measured photocurrent beat-note signals.

the function of a DPLL as a phase-meter core is summarized. The standard application of four independent DPLLs to the segments of a QPD is described in Sec. III and the proposed scheme in Sec. IV. A noise analysis of the new technique, with a comparison against the conventional method, is reported in Sec. V, followed by a conclusion in Sec. VI.

\section{PHASE MEASUREMENT WITH DIGITAL PHASE-LOCKED LOOPS}

The principle of a DPLL is to generate a digital sine wave in a numerically controlled oscillator (NCO) and make it track the incoming sinusoidal beat-note signal in frequency and phase. After appropriate signal conditioning, the incoming signal is first digitized in an analogto-digital converter (ADC) and all remaining processing happens in the digital domain, typically implemented in a field-programmable gate array (FPGA) for the tracking part (see Fig. 2).

The NCO consists of a phase-increment register (PIR) that represents the instantaneous signal frequency, a phase accumulator (PA), which holds the integral of the frequency, i.e., the instantaneous phase, and a look-up table (LUT) that converts the phase into a sine wave and optionally also a cosine wave. The ADC and all digital blocks are driven synchronously from a common clock, which sets the reference for any single phase measurement.

In order to make the NCO sine signal track the incoming signal, both are mixed in a multiplier that acts as a phase detector and the phase deviation thus measured is used as error signal in a servo loop. When the loop is closed and locked, both the incoming and the NCO sine signal have the same frequency and their phase is shifted by $90^{\circ}$, such that their product, the error signal, has a zero average. The incoming and NCO sine signals are said to be "in quadrature" (denoted by " $Q$ "). An optional second branch multiplies the incoming signal with a digital cosine signal, which is then "in phase" ("I") and which can be used to

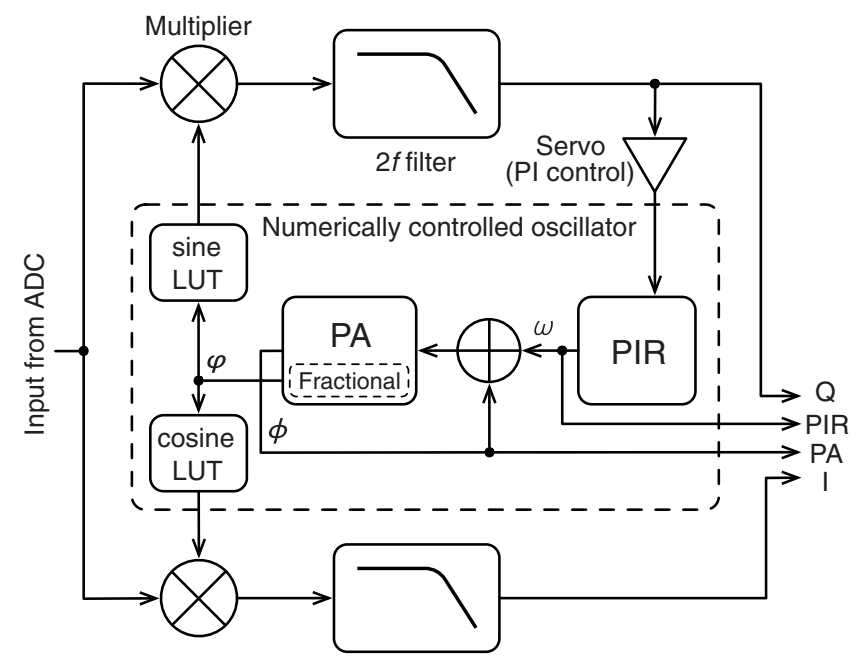

FIG. 2. The functional blocks of a DPLL. The input signal from the ADC is separately mixed with a sine and a cosine delivered by the NCO, yielding the "in quadrature" $Q$ and "in phase" $I$ signals. The second harmonic of the beat-note frequency $2 f$ is suppressed by low-pass filtering these signals. The $Q$ signal is then used as error signal for the servo. The output of the proportional-integral (PI) controller gives the instantaneous signal frequency and is stored in the PIR. This is then integrated and stored in the PA, which is fed to the sine and cosine look-up tables (LUT) that close the loop.

measure the amplitude of the incoming signal. Low-pass filters after the mixers suppress the second harmonic of the signal frequency (" $2 f$ "), a by-product of the multiplication, in order to prevent it from circulating around the loop in an undesired nonlinear process. The primary achievement of such a DPLL is that the frequency and phase now exist in digital form in the PIR and PA registers, respectively, within the NCO, from where they can be directly read out.

More specifically, the PIR holds the instantaneous signal frequency $\omega$ in units of cycles per clock period, with $0<\omega<0.5$. It slowly varies as the input signal frequency changes. It is integrated in the PA, which always has a fractional part $\varphi$, with $0 \leq \varphi<1$ cycles, which is used by the LUTs. It follows a rapid sawtooth function. In most cases, the integer number of cycles (wavelengths) must also be tracked. This can be achieved by including extra bits in the PA that represent the integer number of cycles. We denote that extended PA by $\Phi$, with

$$
\varphi=\Phi \bmod 1
$$

simply being the fractional part of $\Phi$. The total phase $\Phi$ is an ever-increasing ramp. Instead of using extra bits in the PA, the total phase can, in principle, also be reconstructed by integrating $\omega$ externally.

Among the many performance parameters of a DPLL, the most important here is the ability to continuously track 
the input signal without cycle slips, i.e., integer errors in $\Phi$ caused by excess noise in the input signal. That robustness can be optimized by carefully adapting the servo gain to the signal dynamics and enhancing the signal-to-noise ratio (SNR) of the input signal [11,17,23].

\section{DIFFERENTIAL WAVEFRONT SENSING WITH DPLL PHASE METERS}

If a QPD is used in order to implement DWS, the standard procedure is to connect four independent DPLLs to the four segments $A, B, C$, and $D$ of the QPD. We call their error signals $E_{A} \ldots E_{D}$, their PA register contents $\Phi_{A} \ldots \Phi_{D}$, and the fractional parts of the latter $\varphi_{A} \ldots \varphi_{D}$. They are combined to form the output signals:

$$
\begin{aligned}
x & =\frac{\Phi_{A}+\Phi_{B}+\Phi_{C}+\Phi_{D}}{4}, \\
\alpha & =\frac{\varphi_{A}-\varphi_{B}+\varphi_{C}-\varphi_{D}}{2}, \\
\beta & =\frac{\varphi_{A}+\varphi_{B}-\varphi_{C}-\varphi_{D}}{2},
\end{aligned}
$$

where $x$ represents the length signal in units of the laser wavelength and $\alpha$ and $\beta$, reduced to the range -0.5 cycles $\leq \alpha, \beta \leq+0.5$ cycles, represent the horizontal and vertical tilt angles between the wavefronts, scaled by a huge factor that depends on the beam geometry [24]. One more independent linear combination of the segment phases can be formed, which we call the ellipticity. It is rarely used but we include it here in anticipation of the next steps:

$$
\varepsilon=\frac{\varphi_{A}-\varphi_{B}-\varphi_{C}+\varphi_{D}}{2} .
$$

The realization of this scheme is illustrated in Fig. 3. Combination of the signals according to Eqs. (2)-(4) is not the only possibility [25]. In the LISA Pathfinder mission $[1,4,18]$, for example, $x$ was computed as the argument of a complex vector formed by adding the complex amplitudes from the four segments. This differs from Eq. (2) in that the segment contributions are weighted with their respective beat-note amplitudes. Which of these methods is preferable with respect to, e.g., tilt-to-length cross-coupling is still a subject of investigation. If the segment amplitudes are, however, also measured with the " $I$ " branch of the DPLL and recorded, the results can be converted into each other in postprocessing.

This standard scheme has been successfully used in many applications, notably for the continuous active beam pointing over $200-\mathrm{km}$ separation of the intersatellite interferometer in the LRI on GRACE Follow-On [16]. There, and in other applications such as LISA, it is, however, not an optimal method:

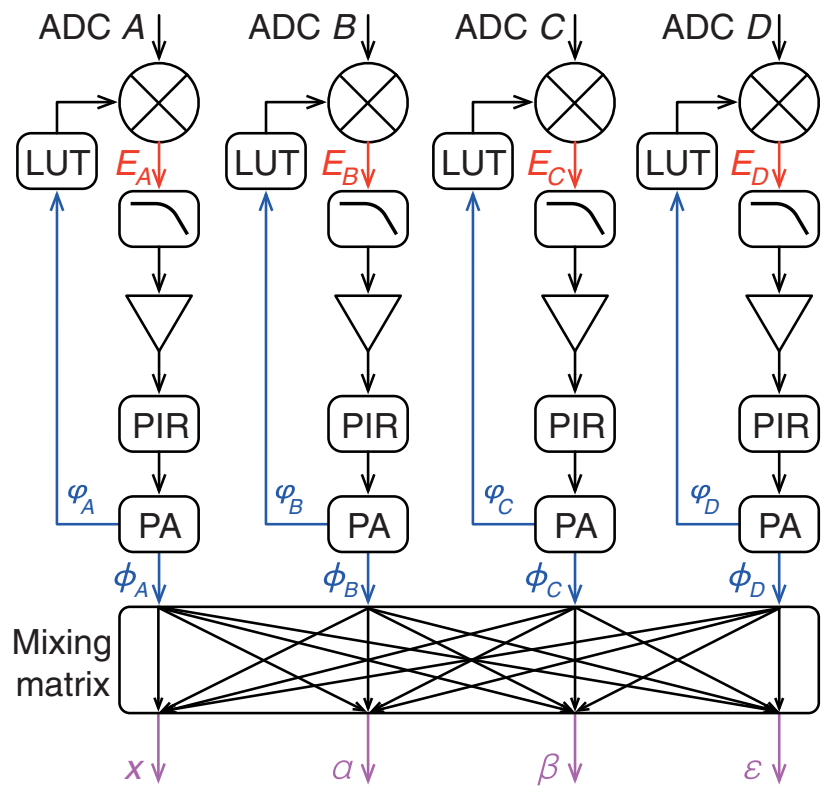

FIG. 3. The conventional readout scheme for a quadrant photodiode. Each DPLL processes 1/4 of the total signal power, which nevertheless has the full dynamics and noise of the length signal.

(a) The beam divergence over the long distance together with the finite aperture of the receiving telescope results in a received beam with extremely low power (in the order of $100 \mathrm{pW}$ to a few nanowatts) and consequently in a poor SNR in the heterodyne beat note to be tracked, which limits the achievable robustness of the tracking loops, each of which uses only $1 / 4$ of the total signal power.

(b) The length signal $x$ has a much higher dynamic range than the angular signals. The former contains the Doppler shift due to the relative spacecraft motion (typically moving at speeds of 8 meters per second or less, corresponding to Doppler shifts of a few megahertz for a wavelength of about $1 \mu \mathrm{m}$ ), as well as common-mode noise sources such as laser-frequency noise, which largely cancel in the other three angular signals $\alpha, \beta$, and $\varepsilon$.

(c) Nevertheless, each of the four standard tracking loops contains the full-length signal, which sets stringent requirements on their loop bandwidths. The resulting open-loop gains are in general not optimal for the much quieter angular signals. The same holds for the $2 f$ filters.

(d) The integer number of cycles, which physically exists only once for each pair of interfering beams, is represented four times in $\Phi_{A} \ldots \Phi_{D}$. These values should represent the same number of integer cycles and differ only by the small quantities $\alpha, \beta$, and $\varepsilon$. If, however, a cycle slip occurs in only some of the four segments, the length signal $x$ computed by Eq. (2) is easily messed up and therefore the measurement is degraded. 


\section{ALTERNATIVE ARCHITECTURE FOR TRACKING LENGTH AND DWS SIGNALS WITH DPLL PHASE METERS}

In order to overcome the above limitations, here we propose an alternative loop topology, in which the four servo loops do not act on the four segments, but on $x, \alpha, \beta$, and $\varepsilon$ instead. Error signals for these loops can be obtained from equations similar to Eqs. (2)-(5):

$$
\begin{aligned}
& E_{x}=\frac{E_{A}+E_{B}+E_{C}+E_{D}}{4}, \\
& E_{\alpha}=\frac{E_{A}-E_{B}+E_{C}-E_{D}}{2}, \\
& E_{\beta}=\frac{E_{A}+E_{B}-E_{C}-E_{D}}{2}, \\
& E_{\varepsilon}=\frac{E_{A}-E_{B}-E_{C}+E_{D}}{2} .
\end{aligned}
$$

The final registers of the four loops track $x, \alpha, \beta$, and $\varepsilon$, which directly represent the desired final output of the phase meter. The segment phases, which are still needed for the multiplicative phase detectors, can be easily obtained by inverting Eqs. (2)-(5):

$$
\begin{aligned}
& \varphi_{A}=x+\frac{\alpha+\beta+\varepsilon}{2}, \\
& \varphi_{B}=x+\frac{-\alpha+\beta-\varepsilon}{2}, \\
& \varphi_{C}=x+\frac{\alpha-\beta-\varepsilon}{2}, \\
& \varphi_{D}=x+\frac{-\alpha-\beta+\varepsilon}{2},
\end{aligned}
$$

where only the fractional part of $x$ needs to be used and the results are reduced to the range $0 \ldots 1$ cycles. The realization of this scheme is illustrated in Fig. 4.

Only $E_{x}$ carries the burden of the large dynamic range of the length signal, e.g., due to the Doppler effect in the orbital motion. It uses the combined signal from all four segments, each of which has been coherently demodulated with individually optimized phases. For noise sources that are uncorrelated among QPD segments, such as shot noise or electronic noise, this yields a 6-dB improvement in the SNR, which makes the loop more robust against cycle slips. Now, there is only one full NCO in the system, which produces the phase ramp $\Phi$ and its sawtoothlike fractional part $\varphi$ and, in particular, only one register $\Phi$ that keeps track of the integer number of cycles, which better maps the physical reality than having four registers $\Phi_{A} \ldots \Phi_{D}$.

The signals in $E_{\alpha}, E_{\beta}$, and $E_{\varepsilon}$, on the other hand, have a much smaller dynamic range, since both the length signal as well as many correlated noise contributions, such as laser-frequency noise or residual-intensity noise, largely

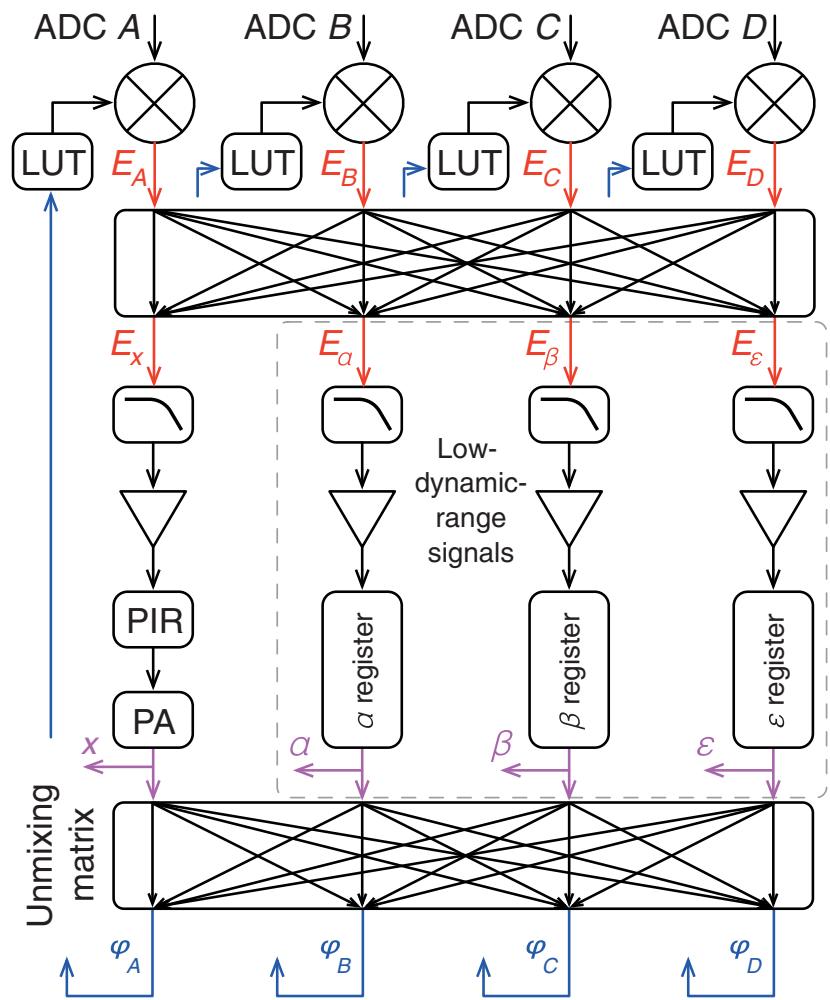

FIG. 4. A proposed DPLL readout scheme for a quadrant photodiode. The error signals from the four QPD segments $E_{A} \ldots E_{D}$ are used to compute DWS error signals $E_{x} \ldots E_{\varepsilon}$. These are then fed to four dedicated DPLLs. The four outputs $x \ldots \varepsilon$, now corresponding to physically meaningful observables, are then reverted back into the phase signals $\varphi_{A} \ldots \varphi_{D}$ and used for closing the loop. Only one DPLL deals with the length signal, while the other three loops handle signals of much smaller dynamic range (encircled). Each servo can hence be individually tuned to the particular dynamical behavior of the signal being tracked.

cancel out. Moreover, the angular degrees of freedom typically change much more slowly than, e.g., spacecraft separation in applications such as the LISA mission or the GRACE Follow-On mission. The outputs of their servo loops are slowly varying numbers with a range that can be limited to, e.g., -0.5 cycles $\leq \alpha, \beta, \varepsilon \leq+0.5$ cycles. If physical constraints exist, e.g., from the optical layout, that further limit their actually achievable range, such constraints can easily be implemented by restricting the range of values that the respective registers are allowed to assume, further increasing the robustness. In particular, the ellipticity $\varepsilon$ will be almost constant in many cases, since it mainly depends on geometrical imperfections of the laser beams.

Each loop can now be individually optimized for the dynamical behavior and noise characteristics of the corresponding signal. This concerns not only the servo-loop bandwidths but also the $2 f$ filters, which can now be made more efficient for the $\alpha, \beta$, and $\varepsilon$ loops. 
Going one step further, one could even think of using an optimized Kalman-type estimator for $\alpha, \beta$, and $\epsilon$ instead of a simple PI controller. The angles $\alpha$ and $\beta$ typically represent the pitch and yaw of either the spacecraft or a test mass and their dynamics consist of noise and commanded actuator signals. The latter are known and could even be used in a feedforward path to further reduce the burden on these filters.

At a first glance, it may seem that this optical readout system could produce fundamentally different outputs, since the mixing matrix that implements Eqs. (6)-(9) acts on the error signals, which are weighted with the signal amplitudes in the individual segments $A \ldots D$, whereas Eqs. (2)-(5) act on the phases that have been stripped of the amplitude information. Further analysis shows, however, that this is not the case and that the proposed system produces the same outputs as the conventional one, at least if the servo loops have enough gain and sufficient integrator stages to keep all error signals close to zero. In that case, Eqs. (6)-(9) imply that the segment error signals $E_{A} \ldots E_{D}$ are zero as well, which leads to the same outputs as in the conventional architecture.

The proposed architecture has been verified to produce the same outputs as the conventional scheme by means of computer simulations and electrical tests [26]. For example, using a dynamical DPLL model implemented in MATLAB SIMULINK, we show that, in the absence of noise and with identical loop parameters, the conventional and the proposed architectures are mathematically equivalent (Fig. 5).

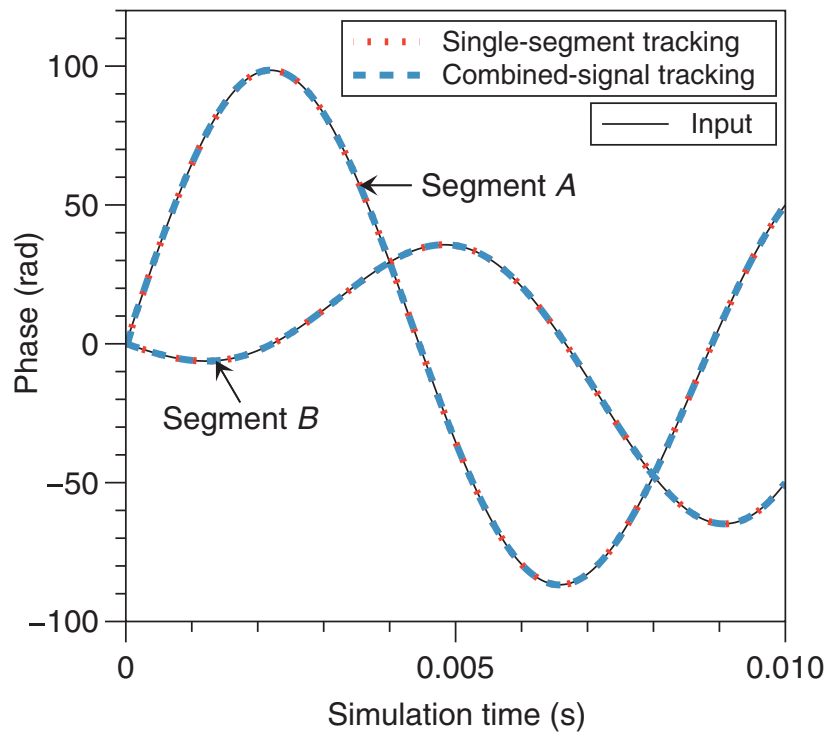

FIG. 5. Simulation of the conventional (single-segment tracking) and the proposed (combined-signal tracking) DWS architectures with DPLL readout, showing the successful tracking of two segments of a quadrant photodiode. Given the same input with no noise and identical loop parameters, the two architectures produce the same output.
Initial tests of the proposed architecture using optical signals are performed in a simple Mach-Zehnder interferometer. The light sources are two Nd:YAG nonplanar ring oscillator (NPRO) lasers, phase locked to each other with a 9-MHz offset with separate electronics. The signals are recorded with an (In, Ga)As QPD of $1 \mathrm{~mm}$ diameter and are converted to voltages with optical-amplifier-based transimpedance amplifiers with $20-\mathrm{MHz}$ bandwidth. The phase meter operates at $80 \mathrm{MHz}$ clock frequency and uses the hardware that is described in Ref. [[17], Ch. 9]. Other than implementing the mixing matrices shown in Fig. 4, the loop parameters are not changed between the conventional and the proposed schemes. The initial measurements confirm that the scheme works functionally and is able to determine correct length and DWS signals. The length loop in the proposed scheme can indeed handle signals of roughly half the amplitude $(-6 \mathrm{~dB})$ compared to the standard scheme, both in acquiring the signal and in tracking without cycle slipping, when the artificially increased noise floor remains unchanged or with the same signal amplitude and $6 \mathrm{~dB}$ more noise. Further experiments are being prepared using a LISA-representative test bed [27].

\section{NOISE INVESTIGATIONS}

We analyze the proposed technique in the context of a heterodyne interferometer in the presence of relativeintensity noise (RIN) from the laser sources, which is uncorrelated among the interfering laser beams but correlated among QPD segments; and photon shot noise, which is uncorrelated among QPD segments. This analysis can be easily scaled to include any number of correlated and uncorrelated noise sources. Let $P_{j k}$ denote the optical power impinging in segment $k \in\{A, B, C, D\}$ of the photodiode located in port $j \in\{A, B\}$ of the interferometer consisting of a pair of QPDs mounted at the output ports of a beam combiner (BS) and let $E_{1}$ and $E_{2}$ denote the onaxis complex amplitudes of the interfering fields (Fig. 6). Upon combination, the superposed fields have complex amplitudes given by

$$
\begin{aligned}
& \text { Port A : } E_{A}=\rho E_{1}+\tau E_{2}, \\
& \text { Port B : } E_{B}=\rho E_{1}+\tau e^{i \pi} E_{2}=\rho E_{1}-\tau E_{2},
\end{aligned}
$$

where $\rho$ and $\tau$ are the beam splitter's amplitude reflection and transmission coefficients, respectively. The photodiode signal in the quadrant labeled by $\{j, k\}=$ \{port, segment $\}$ is given by $C_{j k}=c_{j k} P_{j k}$, where $c_{j k}$ is a constant determined by the analog processing of the signal. The optical power $P_{j k}$ is calculated as

$$
P_{j k}=\iint\left|E_{j}\right|^{2} d S_{j k}
$$

where $d S_{j k}$ is a surface element in segment $k$ of the photodiode in port $j$. For example, for a segment in port $A$, we 


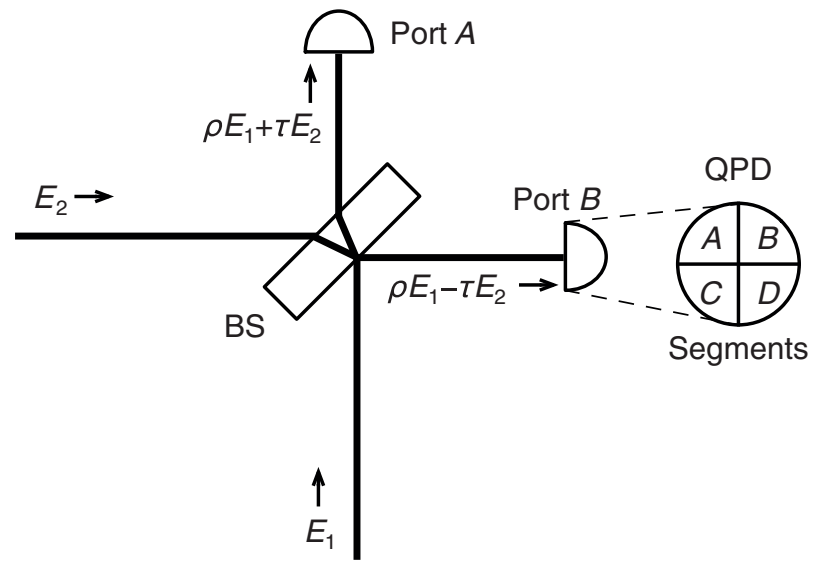

FIG. 6. A diagram showing the interferometer being considered. $E_{1}$ and $E_{2}$ interfere at a beam splitter (BS) and a quadrant photodiode is placed at each of the output ports.

have

$$
\begin{aligned}
P_{A k}= & \rho^{2} \iint\left|E_{1}\right|^{2} d S_{A k}+\tau^{2} \iint\left|E_{2}\right|^{2} d S_{A k} \\
& +\rho \tau\left(\iint E_{1} E_{2}^{*} d S_{A k}+\iint E_{1}^{*} E_{2} d S_{A k}\right) .
\end{aligned}
$$

Here, we introduce the definition of the overlap integral as

$$
\sqrt{\eta_{j k}} e^{i \phi_{j k}} \equiv \frac{\iint E_{1} E_{2}^{*} d S_{j k}}{\left(\iint\left|E_{1}\right|^{2} d S_{j k} \iint\left|E_{2}\right|^{2} d S_{j k}\right)^{1 / 2}},
$$

where $\eta_{j k}$ is the heterodyne efficiency and $\phi_{j k}=2 \pi f_{\text {het }} t-$ $\psi_{j k}$ is the phase of the superposed optical fields, with $f_{\text {het }}$ the heterodyne frequency (i.e., the beat-note frequency between $E_{1}$ and $\left.E_{2}\right)$ and $\psi_{j k}$ the interferometric phase. Recasting Eq. (17) in terms of $\eta_{A k}$ and $\phi_{A k}$ yields

$$
\begin{aligned}
P_{A k} & =\frac{1}{4}\left(\rho^{2} P_{1}+\tau^{2} P_{2}+\rho \tau \sqrt{\eta_{A k} P_{1} P_{2}}\left[e^{i \phi_{A k}}+e^{-i \phi_{A k}}\right]\right) \\
& =\frac{1}{4}\left(\rho^{2} P_{1}+\tau^{2} P_{2}+2 \rho \tau \sqrt{\eta_{A k} P_{1} P_{2}} \cos \phi_{A k}\right), \text { (19) }
\end{aligned}
$$

where $P_{1}$ and $P_{2}$ represent the instantaneous power in the interfering beams and we make the assumption that the beams are well centered in the detectors such that the power is equally distributed over the four QPD segments. This is a reasonable assumption for well-aligned interferometers, as well as for interferometers employing imaging systems to minimize beam walk at the detectors. RIN is a multiplicative noise source and it can be modeled as

$$
\begin{aligned}
& P_{1}=\bar{P}_{1}+\delta P_{1}=\bar{P}_{1}\left(1+r_{1}\right), \\
& P_{2}=\bar{P}_{2}+\delta P_{2}=\bar{P}_{2}\left(1+r_{2}\right),
\end{aligned}
$$

where $\bar{P}_{1}$ and $\bar{P}_{2}$ represent the average power in the interfering fields and RIN is defined as the power noise normalized to the average power, $r_{i}=\delta P_{i} / \bar{P}_{i}$ with $i=\{1,2\}$. By making the appropriate substitutions in Eq. (19), we obtain

$$
\begin{aligned}
P_{A k}= & \frac{1}{4} \rho^{2} \bar{P}_{1}\left(1+r_{1}\right)+\frac{1}{4} \tau^{2} \bar{P}_{2}\left(1+r_{2}\right) \\
& +\frac{1}{2} \rho \tau \sqrt{\eta_{A k} \bar{P}_{1} \bar{P}_{2}\left(1+r_{1}\right)\left(1+r_{2}\right)} \cos \phi_{A k} \\
\approx & \frac{1}{4}\left(\rho^{2} \bar{P}_{1}+\tau^{2} \bar{P}_{2}+\rho^{2} \bar{P}_{1} r_{1}+\tau^{2} \bar{P}_{2} r_{2}\right) \\
& +\frac{1}{2} \rho \tau\left(1+\frac{r_{1}+r_{2}}{2}\right) \sqrt{\eta_{A k} \bar{P}_{1} \bar{P}_{2}} \cos \phi_{A k},
\end{aligned}
$$

where we use $\sqrt{1+r} \approx 1+r / 2$, and we neglect the small cross term $r_{1} r_{2}$. The term $\frac{1}{4}\left(\rho^{2} \bar{P}_{1}+\tau^{2} \bar{P}_{2}\right)$ is the dc coupling and the term $\frac{1}{4}\left(\rho^{2} \bar{P}_{1} r_{1}+\tau^{2} \bar{P}_{2} r_{2}\right)$ is known as $1 f$ RIN and is additive noise. The beat-note signal is mixed with $2 f$-RIN proportional to $\left(r_{1}+r_{2}\right) / 2$. The $2 f$-RIN coupling to the interferometric phase is independent of the beam powers and the heterodyne efficiency and it is a maximum for $\bar{P}_{1}=\bar{P}_{2}$, where the $2 f$-RIN coupling is $1 / 2$ that of the corresponding $1 f$-RIN coupling. Since the total RIN coupling is the root sum square of the two contributions, neglecting $2 f$-RIN induces only a small relative error of a maximum of $1-1 / \sqrt{1+(1 / 2)^{2}} \approx 10 \%$, which becomes much less significant if the beams have different powers (e.g., it is less than $3 \%$ for $\bar{P}_{1} / \bar{P}_{2}=10$ ). To simplify our analysis, we disregard this small term. The photodiodesegment signals can then be written as the combination of three terms,

$$
C_{j k}=c_{j k} P_{j k}=a_{j k}+r_{j k}+b_{j k},
$$

where $a_{j k}$ is the dc coupling of segment $k$ in port $j, r_{j k}$ is the $1 f$-RIN coupling, and $b_{j k}$ is the beat-note signal. For example, for port $A$,

$$
\begin{aligned}
a_{A k} & =\frac{1}{4} c_{A k}\left(\rho^{2} \bar{P}_{1}+\tau^{2} \bar{P}_{2}\right), \\
r_{A k} & =\frac{1}{4} c_{A k}\left(\rho^{2} \bar{P}_{1} r_{1}+\tau^{2} \bar{P}_{2} r_{2}\right), \\
b_{j k} & =\frac{1}{2} \rho \tau c_{j k} \sqrt{\eta_{j k} \bar{P}_{1} \bar{P}_{2}} \cos \phi_{j k} .
\end{aligned}
$$

To characterize the resulting interferometric phase error, we calculate the inverse carrier-to-noise $(\mathrm{C} / \mathrm{N})$ density:

$$
\widetilde{\psi}_{j k}=\frac{\text { Noise ASD in } C_{j k}}{\text { Signal RMS in } C_{j k}}=\frac{\widetilde{\Delta C_{j k}}}{\mathcal{R}\left\{b_{j k}\right\}},
$$

where $\widetilde{\psi}_{j k}$ denotes the amplitude spectral density (ASD) of the interferometric phase, $\mathcal{R}\left\{b_{j k}\right\}$ is the root-mean-square 
(rms) value of the beat-note signal, and $\widetilde{\Delta C}$ is is the ASD of the noise in the photodiode-segment signal,

$$
\widetilde{\Delta C_{j k}}=\sqrt{\tilde{s}_{j k}^{2}+\tilde{r}_{j k}^{2}},
$$

where $\tilde{s}_{j k}$ is the ASD of the shot-noise coupling and $\tilde{r}_{j k}$ is the ASD of the $1 f$-RIN coupling. The shot noise of the photodetection is white noise affecting all frequencies and typically it is the limiting noise source at high frequency. The ASD of shot noise is given for each detector segment as

$$
\tilde{s}_{j k}=\sqrt{2 q_{e} a_{j k}},
$$

where $q_{e}$ is the electron charge. Shot noise adds quadratically with $1 f$-RIN to the total phase noise. The $1 f$-RIN coupling ASD for a segment in port $A$ is given by

$$
\tilde{r}_{A k}=\frac{1}{4} c_{A k}\left(\rho^{2} \bar{P}_{1} \tilde{r}_{1}+\tau^{2} \bar{P}_{2} \tilde{r}_{2}\right) .
$$

The addition in Eq. (30) is performed linearly if the RIN in $E_{1}$ and $E_{2}$ is correlated and quadratically otherwise. The rms value of the signal is

$$
\mathcal{R}\left\{b_{j k}\right\}=\frac{1}{4} \rho \tau c_{j k} \sqrt{2 \eta_{j k} \bar{P}_{1} \bar{P}_{2}},
$$

where we have used the definition of the rms value of a function of period $T$ :

$$
\mathcal{R}\{f(t)\}=\sqrt{\frac{1}{T} \int_{0}^{T}[f(t)]^{2} d t .}
$$

The phase noise for a segment in port $A$ considering $1 f$ RIN coupling only is given by

$$
\widetilde{\psi}_{A k}=\frac{\rho^{2} \bar{P}_{1} \tilde{r}_{1}+\tau^{2} \bar{P}_{2} \tilde{r}_{2}}{\rho \tau \sqrt{2 \eta_{A k} \bar{P}_{1} \bar{P}_{2}}} .
$$

The phase noise due to shot noise only is given by

$$
\widetilde{\psi}_{A k}=\frac{2}{\rho \tau} \sqrt{\frac{q_{e}\left(\rho^{2} \bar{P}_{1}+\tau^{2} \bar{P}_{2}\right)}{c_{A k} \eta_{A k} \bar{P}_{1} \bar{P}_{2}}} .
$$

Each QPD segment "sees" $1 / 4$ of the signal and 1/4 of the $1 f$-RIN and hence has the same phase error associated with $1 f$-RIN coupling as a single-element photodiode
(SEPD) with four times the surface area. However, each segment sees up to $1 / 2$ of the shot noise as the SEPD, resulting in up to twice the phase error associated with shot noise or with any source of noise that is uncorrelated among segments as the SEPD. The total phase noise in port $A$ of the interferometer is given by

$$
\widetilde{\psi}_{A k}=\frac{\sqrt{\tilde{s}_{A k}^{2}+\tilde{r}_{A k}^{2}}}{\frac{1}{4} \rho \tau c_{A k} \sqrt{2 \eta_{A k} \bar{P}_{1} \bar{P}_{2}}},
$$

where we have assumed that the RIN in $E_{1}$ and $E_{2}$ is uncorrelated, so that $\tilde{r}_{1}$ and $\tilde{r}_{2}$ add quadratically. In the conventional DWS architecture, the PLL acts on the signals $C_{j k}$ of the individual QPD segments and thus the performance is limited by the phase noise given in Eq. (35). In the proposed DWS architecture, the $C_{j k}$ signals are first combined linearly and the PLL acts on the linear combinations. The shot noise is uncorrelated among segments and adds quadratically, whereas $1 f$-RIN is correlated and adds linearly.

For example, for tracking the length signal, we combine the four QPD quadrants in port $A$ as

$$
C_{A(A+B+C+D)}=\sum_{k=A}^{D} C_{A k}=\sum_{k=A}^{D} a_{A k}+\sum_{k=A}^{D} r_{A k}+\sum_{k=A}^{D} b_{A k} .
$$

We omit the factor $1 / 4$ in the signal combination [see Eq. (6)], since it affects the noise ASD and the beat-note signal rms value of the combined signal equally and therefore has no impact on the SNR of the tracked signal. Note that in this case the PLL does not track the phase of the beat-note signal $E_{A}=\rho E_{1}+\tau E_{2}$ but instead tracks the phase of the beat-note signal that results from adding the four QPD segments $A, B, C$, and $D$. The noise ASD of the combined signal is given by

$$
\widetilde{\Delta C}_{A(A+B+C+D)}=\left(\sum_{k=A}^{D} \tilde{s}_{A k}^{2}+\left[\sum_{k=A}^{D} \tilde{r}_{A k}\right]^{2}\right)^{1 / 2}
$$

and the rms value of the signal is given by

$$
\mathcal{R}\left\{\sum_{k=A}^{D} b_{A k}\right\}=\frac{\rho \tau \sqrt{\bar{P}_{1} \bar{P}_{2}}}{2 \sqrt{2}}\left(\sum_{k_{1}, k_{2}=A}^{D} c_{A k_{1}} c_{A k_{2}} \sqrt{\eta_{A k_{1}} \eta_{A k_{2}}}\right)^{1 / 2}=\frac{\rho \tau \sqrt{\bar{P}_{1} \bar{P}_{2}}}{2 \sqrt{2}}\left(\sum_{k=A}^{D} c_{A k}^{2} \eta_{A k}+2 \sum_{A \leq k_{1}<k_{2} \leq D} c_{A k_{1}} c_{A k_{2}} \sqrt{\eta_{A k_{1}} \eta_{A k_{2}}}\right)^{1 / 2} .
$$


where $k_{1}, k_{2} \in\{A, B, C, D\}$. Equations (37) and (38) can be substituted into Eq. (27) to obtain the total noise of the combined signal of the four QPD segments. The resulting expression is quite long. By assuming identical analog signal processing $c_{A k_{1}}=c_{A k_{2}}=c_{A}$ and segment heterodyne efficiencies $\eta_{A k_{1}}=\eta_{A k_{2}}=\eta_{A}$, we obtain the much simpler expression

$$
\begin{aligned}
& \widetilde{E}_{A(A+B+C+D)} \\
& \quad \approx \frac{1}{\rho \tau} \sqrt{\frac{2 q_{e}\left(\rho^{2} \bar{P}_{1}+\tau^{2} \bar{P}_{2}\right)+c_{A}\left(\rho^{4} \bar{P}_{1}^{2} \tilde{r}_{1}^{2}+\tau^{4} \bar{P}_{2} \tilde{r}_{2}^{2}\right)}{2 c_{A} \eta_{A} \bar{P}_{1} \bar{P}_{2}}} .
\end{aligned}
$$

The combined signal has the same level of $1 f$-RIN coupling as in the single-segment case [Eq. (33)] but lower shot-noise coupling. The shot noise in Eq. (39) is $1 / 2$ that of the single-segment case [Eq. (34)].

Equation (35) (i.e., the phase noise in single-segment tracking) and Eq. (39) can be compared to assess the improvement due to the proposed technique:

$$
\frac{\widetilde{E}_{A(A+B+C+D)}}{\widetilde{\psi}_{A A}} \approx \sqrt{1-\frac{12 q_{e}\left(\bar{P}_{1}+\bar{P}_{2}\right)}{16 q_{e}\left(\bar{P}_{1}+\bar{P}_{2}\right)+c_{A}\left(\bar{P}_{1}^{2} \tilde{r}_{1}^{2}+\bar{P}_{2}^{2} \tilde{r}_{2}^{2}\right)}} .
$$

It can now readily be seen that the proposed technique provides a maximum improvement of $6 \mathrm{~dB}$ over the traditional scheme:

$$
\begin{aligned}
20 & \times \log \left(\frac{\widetilde{E}_{A(A+B+C+D)}}{\widetilde{\psi}_{A A}}\right) \approx 20 \times \log \left(\sqrt{1-\frac{3}{4}}\right) \\
& \approx-6 \mathrm{~dB} .
\end{aligned}
$$

When tracking the interferometric phase signal from a single segment of a QPD, the PLL "sees" $1 / 4$ of the signal rms value and $1 / 4$ of the $1 f$-RIN but up to $1 / 2$ of the shot noise, as a SEPD. This results in up to twice the shot-noise-induced phase error as when tracking the phase signal of the SEPD. To take full advantage of the QPD, in the proposed architecture the PLL is applied not to the individual QPD segments but to the linear combinations of these signals [Eqs. (6)-(9)]. In this way, the tracked signals will have the same level of correlated noise coupling, such as $1 f$-RIN, as in single-segment tracking but as little as half as much uncorrelated noise coupling, such as shot noise, resulting in a similar noise performance overall as when tracking the signal from a SEPD of the same size as the QPD. This is in addition to other advantages, such as being able to adjust the loop gains and filter parameters individually for length and angular signals.

In the LISA long-arm interferometer, one field is much stronger than the other. In this case, we may simplify

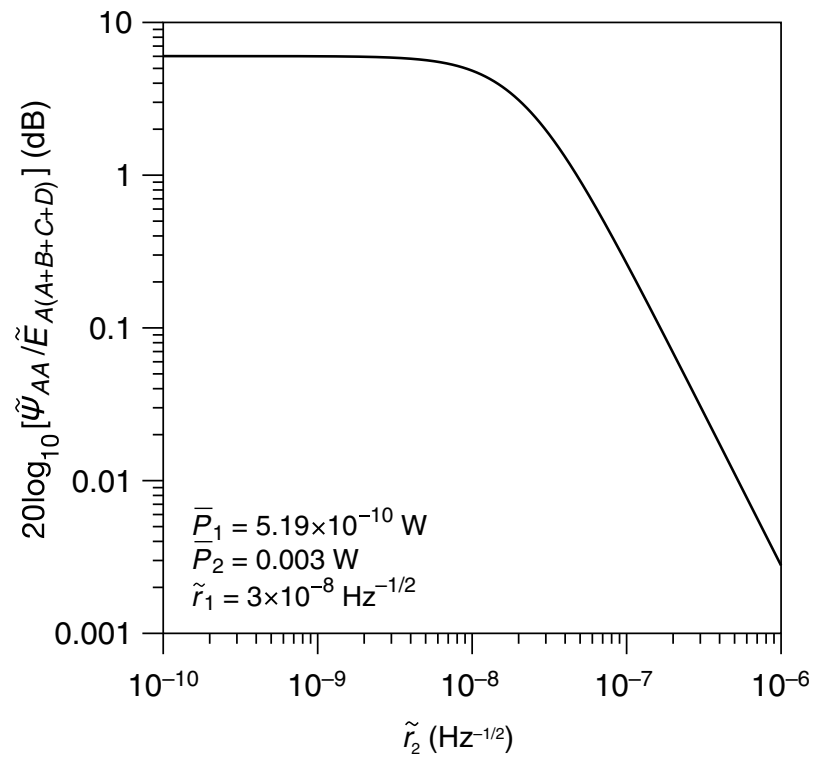

FIG. 7. The improvement due to the proposed scheme over single-segment tracking as a function of the RIN level in the strong field, for an interferometer resembling the LISA long-arm interferometer.

further (i.e., $\bar{P}_{2} \gg \bar{P}_{1}, \bar{P}_{2}^{2} \tilde{r}_{2}^{2} \gg \bar{P}_{1}^{2} \tilde{r}_{1}^{2}$ ):

$$
\frac{\widetilde{E}_{A(A+B+C+D)}}{\widetilde{\psi}_{A A}} \approx \sqrt{1-\frac{12 q_{e} \bar{P}_{2}}{16 q_{e} \bar{P}_{2}+c_{a} \bar{P}_{2}^{2} \tilde{r}_{2}^{2}}}
$$

Figure 7 shows the improvement as a function of the RIN level in the strong field in an interferometer with parameters akin to those of the LISA long-arm interferometer.

A major advantage of the increased SNR in the proposed architecture is the enhanced robustness against alignment errors of the interferometer. This is an issue of great concern in the development of long-baseline space-based observatories, such as LISA, where the unavoidable angular motion of the spacecraft translates into alignment jitter in the interferometer and therefore in a degradation of heterodyne efficiency and a cross-coupling in the longitudinal-path-length measurements known as tilt-tolength (TTL) coupling. Imaging systems can be used to reduce TTL coupling in the interferometer to a great extent $[27,28]$. The imaging systems suppress beam walk in the detector plane, thus leading to the reduction in TTL coupling, but in turn they magnify the beam tilt angle. The angular magnification provided by the imaging systems, in conjunction with that provided by the receiving telescope, means that the phase meter should be able to track signals with relatively poor heterodyne efficiency.

To quantify the improvement due to the proposed technique under the alignment errors of the interferometer, we develop a computer model of the interferometer using 
TABLE I. The simulation parameters. The model assumes identical analog processing of the QPD segments.

\begin{tabular}{lc}
\hline \hline Property & Value \\
\hline $\bar{P}_{1}$ & $519 \mathrm{pW}$ \\
$\bar{P}_{2}$ & $3 \mathrm{~mW}$ \\
$\tilde{r}_{1}$ & $10^{-8} / \sqrt{\mathrm{Hz}}$ \\
$\tilde{r}_{2}$ & $10^{-8} / \sqrt{\mathrm{Hz}}$ \\
$\rho=\tau$ & $1 / \sqrt{2}$ \\
QPD active area diameter & $1 \mathrm{~mm}$ \\
QPD slit width & $20 \mu \mathrm{m}$ \\
Reference-beam waist radius & $0.5 \mathrm{~mm}$ \\
Measurement-beam waist radius & $10 \mathrm{~mm}$ \\
\hline \hline
\end{tabular}

IFOCAD, which is a collection of $\mathrm{C}++$ libraries that provides proven methods for simulating two-beam laser interferometers [24,29]. We simulate the interference between a Gaussian reference beam with typical parameters (LO beam) and a Gaussian beam with a very large waist located at the position of a QPD such that, at this position, it resembles the flat-top beam received by a LISA spacecraft $(\mathrm{Rx}$ beam). This particular simulation method has been verified experimentally in a test bed featuring a flat-top beam on Gaussian-beam interference [27].

We perform a Monte Carlo analysis in which the program computes the heterodyne efficiencies for each of the four segments of the QPD, as the Rx beam is rotated with respect to the center of the detector, so as to mimic the effect of an imaging system that is aligned perfectly and is therefore suppressing the Rx beam walk in the detector plane. The beam tilt angle is sampled from a uniform distribution with increasing amplitude and the tilt direction is sampled from a uniform distribution spanning the entire plane transverse to the optical axis. The program then uses the computed heterodyne efficiencies to calculate the estimated signal error in the conventional [single-segment tracking, Eq. (35)] and the proposed [combined-signal tracking, Eqs. (38) and (37)] architectures. The simulation parameters are gathered in Table I.

As expected, the proposed technique offers an improved SNR throughout the tilt range (Fig. 8). It is therefore able to handle worse absolute beam misalignments and higher residual beam-pointing jitter. This improvement is crucial for a enhanced tolerance of spacecraft attitude-control instabilities prior to acquiring a stable phase locking of a slave laser to a weak received beam, as required for the establishment of an interspacecraft laser link in a lasertransponder configuration. This improved SNR is then specially relevant throughout the initial beat-note acquisition states and reacquisition sequences [30,31], providing more robust control loops for sensing the optical link dynamics.

It is important to note that the noise improvement is achieved in the signals being tracked by each separate PLL instance and not on the length and angular signals. In the conventional scheme, the individual QPD segment signals

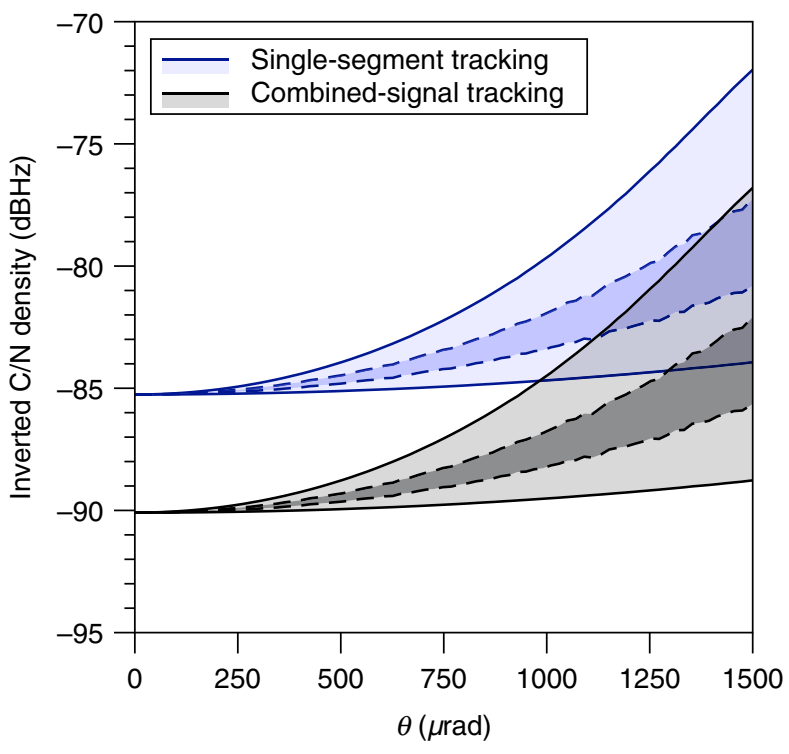

FIG. 8. The inverted carrier-to-noise density of the signals being tracked by the PLL in the conventional (single-segment tracking) and the proposed (combined-signal tracking) architectures, resulting from a Monte Carlo simulation of a misaligned interferometer where the beam tilt angle, as sensed by the detector, is sampled from a uniform distribution with amplitude $\theta$ and the beam-tilt direction is sampled from a uniform distribution spanning the entire plane transverse to the optical axis. The beam tilts with respect to the center of the detector as if it was being imaged to that point by an imaging system. In single-segment tracking, all four QPD segments are taken into consideration in the statistical analysis. The solid lines represent the maxima and minima of the resulting inverted $\mathrm{C} / \mathrm{N}$ density distributions. The dark-shaded regions delimited by dashed lines represent one standard deviation from the mean.

tracked by four PLLs are combined in the next stage to yield length and angular signals with the same level of noise as in the proposed scheme. The advantage of the proposed architecture lies in the enhancement of the tracking loops: the PLLs track signals with a greater SNR, which results in more robust locks with a lower probability of cycle slipping and the added benefit of being able to tune each PLL instance to optimally track its corresponding length or angular signal channel.

\section{CONCLUSION}

We propose a scheme to process the heterodyne beat notes from the four segments of a quadrant photodiode in heterodyne interferometers that use DPLL-based phase meters. It acts on the length signal $x$ and angular signals $\alpha, \beta$, and $\varepsilon$, which directly correspond to physically meaningful parameters. The proposed scheme has advantages in terms of robustness against cycle slips. It allows us to individually optimize the loop gains and filter parameters for length and angular signals, which may also lead to lower 
noise in these outputs. A noise analysis of the proposed architecture is carried out in the context of a heterodyne interferometer in the presence of shot noise and relativeintensity noise. Then, a computer model is used to analyze the degradation of the tracked signals in a misaligned interferometer. The proposed technique is compared against the conventional one to demonstrate a maximum improvement of $6 \mathrm{~dB}$. We expect that this scheme may be an attractive alternative for applications such as LISA or future GRACE Follow-On-like geodesy missions. It can also find applications across multiple disciplines in which high-precision length and angular measurements are desired, notably in inertial sensing of test masses, with applications in fields such as vibration isolation [32] and tests of fundamental physics [33-35].

\section{ACKNOWLEDGMENTS}

The authors acknowledge the support of the German Space Agency, DLR (Grant No. FKZ 50OQ1801); Clusters of Excellence "QuantumFrontiers: Light and Matter at the Quantum Frontier: Foundations and Applications in Metrology" (EXC-2123, Project No. 390837967), and PhoenixD: "Photonics, Optics, and Engineering-Innovation Across Disciplines" (EXC-2122, Project No. 390833453).

[1] M. Armano et al., Beyond the Required LISA FreeFall Performance: New LISA Pathfinder Results down to $20 \mu \mathrm{Hz}$, Phys. Rev. Lett. 120, 061101 (2018).

[2] Klaus Abich et al., In-Orbit Performance of the GRACE Follow-On Laser Ranging Interferometer, Phys. Rev. Lett. 123, 031101 (2019).

[3] A. M. Cruise, D. Hoyland, and S. M. Aston, Implementation of the phasemeter for LISA LTP, Class. Quantum Gravity 22, S165 (2005).

[4] G. Heinzel, V. Wand, A. Garcia, O. P. Jennrich, C. Braxmaier, D. Robertson, K. Middleton, D. Hoyland, A. Rudiger, R. Schilling, U. Johann, and K. Danzmann, The LTP interferometer and phasemeter, Class. Quantum Gravity 21, S581 (2004).

[5] Yu-Rong Liang, Hui-Zong Duan, Hsien-Chi Yeh, and Jun Luo, Fundamental limits on the digital phase measurement method based on cross-correlation analysis, Rev. Sci. Instrum. 83, 095110 (2012).

[6] S. E. Pollack and R. T. Stebbins, Demonstration of the zerocrossing phasemeter with a LISA test-bed interferometer, Class. Quantum Gravity 23, 4189 (2006).

[7] Vinzenz Wand, Felipe Guzman, Gerhard Heinzel, and Karsten Danzmann, in Laser Interferometer Space Antenna, edited by S. M. Merkowitz and J. C. Livas, AIP Conf. Proc. No. 873 (2006), p. 689.

[8] D. Shaddock, B. Ware, P. G. Halverson, R. E. Spero, and B. Klipstein, in Laser Interferometer Space Antenna, edited by S. M. Merkowitz and J. C. Livas, AIP Conf. Proc. No. 873 (2006), p. 654.
[9] Malcolm B. Gray, Terry McRae, Magnus T. L. Hsu, Jan Herrmann, and Daniel A. Shaddock, in 2012 Conference on Lasers and Electro-Optics, Conference on Lasers and Electro-Optics (San Jose, California, USA, 2012).

[10] Oliver Gerberding, Benjamin Sheard, Iouri Bykov, Joachim Kullmann, Juan Jose Esteban Delgado, Karsten Danzmann, and Gerhard Heinzel, Phasemeter core for intersatellite laser heterodyne interferometry: Modelling, simulations and experiments, Class. Quantum Gravity 30, 235029 (2013).

[11] Samuel P. Francis, Timothy T. Y. Lam, Kirk McKenzie, Andrew J. Sutton, Robert L. Ward, David E. McClelland, and Daniel A. Shaddock, Weak-light phase tracking with a low cycle slip rate, Opt. Lett. 39, 5251 (2014).

[12] B. Bachman, G. de Vine, J. Dickson, S. Dubovitsky, J. Liu, W. Klipstein, K. McKenzie, R. Spero, A. Sutton, B. Ware, and C. Woodruff, in 11th International LISA Symposium, Journal of Physics Conference Series, Vol. 840 (Zurich, Switzerland, 2017).

[13] Karsten Danzmann, Lisa Pathfinder Team, and eLisa Consortium, LISA and its pathfinder, Nat. Phys. 11, 613 (2015).

[14] LISA mission L3 proposal.

[15] B. S. Sheard, G. Heinzel, K. Danzmann, D. A. Shaddock, W. M. Klipstein, and W. M. Folkner, Intersatellite laser ranging instrument for the GRACE Follow-On mission, J. Geod. 86, 1083 (2012).

[16] Joint press release by AEI and NASA/JPL, July 2, 2018; paper in preparation.

[17] Oliver Gerberding, Ph.D. thesis, Gottfried Wilhelm Leibniz Universität Hannover (2014).

[18] M. Armano et al., Sub-Femto-g Free Fall for SpaceBased Gravitational Wave Observatories: LISA Pathfinder Results, Phys. Rev. Lett. 116, 231101 (2016).

[19] M. Armano et al., LISA pathfinder platform stability and drag-free performance, Phys. Rev. D 99, 082001 (2019).

[20] Alexander Koch, Josep Sanjuan, Martin Gohlke, Christoph Mahrdt, Nils Brause, Claus Braxmaier, and Gerhard Heinzel, Line of sight calibration for the laser ranging interferometer on-board the GRACE Follow-On mission: Onground experimental validation, Opt. Express 26, 25892 (2018).

[21] E. Morrison, B. J. Meers, D. I. Robertson, and H. Ward, Automatic alignment of optical interferometers, Appl. Opt. 33, 5041 (1994).

[22] E. Morrison, B. J. Meers, D. I. Robertson, and H. Ward, Experimental demonstration of an automatic alignment system for optical interferometers, Appl. Opt. 33, 5037 (1994).

[23] Floyd M. Gardner, Phaselock Techniques (Wiley, New York, 2005).

[24] Gudrun Wanner, Gerhard Heinzel, Evgenia Kochkina, Christoph Mahrdt, Benjamin S. Sheard, Soenke Schuster, and Karsten Danzmann, Methods for simulating the readout of lengths and angles in laser interferometers with Gaussian beams, Opt. Commun. 285, 4831 (2012).

[25] Gudrun Wanner, Soenke Schuster, Michael Troebs, and Gerhard Heinzel, in 10th International LISA Symposium, Journal of Physics Conference Series, Vol. 610 (Florida, USA, 2015). 
[26] Nils Christopher Brause, Ph.D. thesis, Gottfried Wilhelm Leibniz Universität Hannover (2018).

[27] M. Chwalla, K. Danzmann, M. Dovale Álvarez, J. J. Esteban Delgado, G. Fernández Barranco, E. Fitzsimons, O. Gerberding, G. Heinzel, C. J. Killow, M. Lieser, M. Perreur-Lloyd, D. I. Robertson, J. M. Rohr, S. Schuster, T. S. Schwarze, M. Tröbs, G. Wanner, and H. Ward, Optical Suppression of Tilt-to-Length Coupling in the LISA Long-Arm Interferometer, Phys. Rev. Appl. 14, 014030 (2020).

[28] M. Tröbs, S. Schuster, M. Lieser, M. Zwetz, M. Chwalla, K. Danzmann, G. Fernández Barránco, E. D. Fitzsimons, O. Gerberding, G. Heinzel, C. J. Killow, M. PerreurLloyd, D. I. Robertson, T. S. Schwarze, G. Wanner, and H. Ward, Reducing tilt-to-length coupling for the LISA test mass interferometer, Class. Quantum Gravity 35, 105001 (2018).

[29] IFOCAD, http://www.lisa.aei-hannover.de/ifocad/.

[30] Danielle M. R. Wuchenich, Christoph Mahrdt, Benjamin S. Sheard, Samuel P. Francis, Robert E. Spero, John Miller, Conor M. Mow-Lowry, Robert L. Ward, William M. Klipstein, Gerhard Heinzel, Karsten Danzmann, David E. McClelland, and Daniel A. Shaddock, Laser link acquisition demonstration for the GRACE Follow-On mission, Opt. Express 22, 11351 (2014).

[31] Alexander Koch, Ph.D. thesis, Gottfried Wilhelm Leibniz Universität Hannover (2020).

[32] C. M. Mow-Lowry and D. Martynov, A 6D interferometric inertial isolation system, Class. Quantum Gravity 36, 245006 (2019).

[33] S. Schlamminger, K.-Y. Choi, T. A. Wagner, J. H. Gundlach, and E. G. Adelberger, Test of the Equivalence Principle Using a Rotating Torsion Balance, Phys. Rev. Lett. 100, 041101 (2008).

[34] Jun Luo, Qi Liu, Liang-Cheng Tu, Cheng-Gang Shao, LinXia Liu, Shan-Qing Yang, Qing Li, and Ya-Ting Zhang, Determination of the Newtonian Gravitational Constant $G$ with Time-of-Swing Method, Phys. Rev. Lett. 102, 240801 (2009).

[35] Qing Li, Chao Xue, Jian-Ping Liu, Jun-Fei Wu, ShanQing Yang, Cheng-Gang Shao, Li-Di Quan, Wen-Hai Tan, Liang-Cheng Tu, Qi Liu, Hao Xu, Lin-Xia Liu, Qing-Lan Wang, Zhong-Kun Hu, Ze-Bing Zhou, Peng-Shun Luo, Shu-Chao Wu, Vadim Milyukov, and Jun Luo, Measurements of the gravitational constant using two independent methods, Nature 560, 582 (2018). 